Der Chefchemiker der Dominion Iron and Steel Co. A. P. S c ott, ist in die Dienste der General Electrico Co., Schenectady, N. Y., übergetreten.

Dem mit Wahrnehmung des Unterrichts in der konstruktiven und mechanischen $\mathrm{H}$ ü t te n k und e an der technischen Hochschule zu Aachen betrauten Hüttenwerksdirektor $O$. $\mathrm{Sim} \mathrm{mer} \mathrm{s} \mathrm{b} \mathrm{a} \mathrm{ch}$ in Düsseldorf ist das Prädikat "Professor" verliehen worden.

Der Professor für anorganische Chemie an der Universität Bern, Dr. F r i e d b e i $\mathrm{m}$, wird voraussichtlich von seinem Lehramt zurücktreten.

Seinen 70. Geburtstag beging am 1. Januar der ord. Prof- der Physik an der Techn. Hochschule zu Braunschweig, Geh. Hofrat Dr. phil. Heinrich $W$ eber.

Am 20./12. 1908 feierte E. W i 1 d sein 25jähriges Jubiläum als Lehrer der städtischen Chemie. schule in Mülhausen i. E. Er leitet seit 1890 dort den gesamten Unterricht in anorganischer und analytischer Chemie.

In Wiesbaden verstarb am 31./12. Kommerzienrat Heinrich Albert im Alter von 74 Jahren. Der Verstorbene war seit 1894 Vorsitzender des Aufsichtsrates der Chemischen Werke vormals H. u. E. Albert in Amoeneburg b. Biebrich.

\section{Eingelaufene Bücher.}

(Besprechung behalt sich die Redaktion vor.)

Giese, H., Die Verflüssigung d. Luft u. ihre Zerlegung, mit 74 Abb. Leipzig, H. A. L. Degener, 1909.

M 80,-

Landenberger, D., Die besonderen Bestimmungen $\mathrm{d}$. verschiedenen Patentgesetze über d. Patentierung chemischer Erfindungen. Berlin, im Selbstverlag.

\section{Bücherbesprechungen.}

Versuch einer Bewertung chemischer Theorien. Von Dr. A lf r ed Benrath, Privatdozent an der Universität Königsberg. Heidelberg, C. Winter, 1908.

Preis M 1,20

Für den Naturwissenschaftlicher liegt immer die Gefahr nahe, den Theorien und Hypothesen gegenüber den richtigen Standpunkt zu verlieren, sich in die Gedankenwelt so hineinzuleben, daß er sie für Wirklichkeit hält, daß er die Begriffsgebilde mit den Dingen selbst verwechselt. Von Zeit zu Zeit tut da ein Weckruf gut. So will auch vorliegende Schrift zur Kritik mahnen. Was man unter Gesetz, Hypothese, Theorie usw. zu verstehen hat, das setzt der Verf. klar auseinander. Dabei läßt sich natürlich eine gewisse Willkür in der Definition dieser einzelnen Begriffe nicht ausschließen; doch herrscht über die grundlegenden Definitionen wohl ziemlich allgemeine U̇bereinstimmung. Trotzdem kann man oft sehen, wie in Abhandlungen und Büchern die Sache auf den Kopf gestellt wird, indem hypothetische Folgerungen als wirkliche Begründungen von Tatsachen hingestellt werden. Nach einem einleitenden Kapitel über die Terminologie stellt der Verf. in kurzen Zügen die historische Entwicklung der Phlogistontheorie, der Atomhypo- these nebst den mit ihr verbundenen Gesetzen, der Systematik und der Theorie der Lösungen dar und knüpft daran seine kritischen Bemerkungen. In seinen Anschauungen ist er, wie er selber auch im Vorwort sagt, von den Ideen $M$ a chs, $\mathrm{Ost}$ w a $1 \mathrm{ds}$ und $W$ a $1 \mathrm{ds}$ stark beeinflußt. Mag man diesen auoh nioht in allen Punkten zustimmen, der Ruf zur Selbstkritik sollte jedenfalls nicht ungehört verhallen, vor allen bei denjenigen Chemikern, die es lieben, die Formeln als vergrößertes Bild wirklicher Moleküle zu betrachten und sich ans diesen. Molokülen eine Märchenwelt aufzubauen.

\section{Lockemann.}

Vorträge über moderne Chemie für Ing enieure. Gehalten im Österr. Ingenieur- u. ArchitektenVerein in Wien. Komm.-Verlag Wilh. Ernst $\&$ Sohn, Berlin.

Die Vorträge sind in den Jahren 1904 bis 1907 gehalten und bezwecken, die neuen Lehren der modernen Chemie den Chemikern der älteren Schule und den Ingenieuren mitzuteilen und ein Verfolgen derselben $\mathrm{zu}$ ermöglichen.

W. O s t w a 1 d eröffnet den Reigen, es folgen K la u d y, J üptner von Jonstorf $f, P$ meranz, Wegscheider, $S k r a u p$, van't Hoff, Eder, Lunge, Witt, Nernst, $\mathrm{C}$ i a $\mathrm{m}$ i c i a $\mathrm{n}$ und $J \ddot{a} \mathrm{~g} \mathrm{e} \mathrm{r}$, also die ersten Autoritäten auf den einzelnen Gebieten der modernen Chemie. Diese Namen genügen, um den Inhalt der Vorträge zu kennzeichnen, und dürfte wohl niemand, der das Buch studiert hat, dies getan haben, ohne daraus Anregungen und Belehrung in mancherlei Hinsicht erhalten zu haben. Dr. H. Mühlenbein.

Farbenphotographie mit Autochromplatten. Von A. v. H ü b l. Enzyklopädie der Photographie. Halle a. S. 1908.

Der Verf,, eine anerkannte Autorität auf dem Gebiete der Photographie, gibt im ersten Teile des. Buches eine eingehende klare Schilderung der theoretischen Grundlagen der Photographie mit polychromem Raster; im zweiten Teile wird die auf eigene praktische Erfahrung gestützte Verarbeitung. der Autochromplatten behandelt. Jedem, der das interessante $\mathrm{L} \mathrm{u} \mathrm{m}$ i e $\mathrm{r}$ e sche Farbenphotographieverfahren ausübt, wird das $H$ ü b l sche Werkohen ein zuverlässiger Berater sein. König.

Über die Schichtungen bei Diffusionen. Von $R$ a $p h$. F. Li e segang. Düsseldorf 1907.

Verf. untersuchte schon früher die Schichtenbildungen von Silberchromat, welche entstehen, wenn man einen Tropfen $\mathrm{AgNO}_{3}$-Lösung in eine $\mathrm{K}_{2} \mathrm{Cr}_{2} \mathrm{O}_{2}$-haltige Gelatineschicht eindiffundieren läßt Bei Wiederaufnahme der Versuche gelang es zunächst nicht, dieselben Schichtungen wie früher zu erhalten. Schließlich stellte sich heraus, daß die Schichtungen nur dann auftreten, wenn die Gelatinelösung mehrere Tage alt war, oder wenn man frischer Gelatine etwas von einer durch Bakterientätigkeit oder hydrolytische Spaltung verflüssigten Gelatine (Gelaton) zusetzte. Die Arbeit umfaßt sehr zahlreiche Versuche, über die im einzelnen nicht. referiert werden kann. Die Schichtenbildung ist. durch ausgezeichnete Photogramme illustriert.

\section{König.}

Lenz, w. und *. Arends, Hagers Handbuch der pharmazentischen Praxis fir Apotheker, Arzte, 
Drogisten und Medizinalbeamte. Ergänzungsband unter Witwirkung von E $\mathbf{r} \mathbf{n}$ t D a $\mathrm{n} \mathrm{z} 1$ Berlin, M. Pi o rk ow ski-Berlin, A. $\mathrm{S}$ c h m i d t-Geyer, G e or g W e ig e l-Hamburg. Otto Wiegand-Leipzig, Carl Wulff-Buch, Franz Zernick-Steglitz. Mit zahlreichen in den Text gedruckten Figuren. Berlin, Julius Springer, 1908. $820 \mathrm{~S}$. Lexikongröße. Ungeb. M 15; geb. M 17,50 Utberschaut man das Vorleben der beiden Bearbeiter und Herausgeber der "Pharmazeutischen Praxis" des Altmeisters $\mathbf{H}$ a $g$ e $r$, der vorerst. was die praktische Anordnung und die Brauchbarkeit seiner Handbücher kaum seinesgleichen, geschweige einen Besseren gefunden hat, denkt man an des ersteren Arbeiten auf dem Gebiete der Nahrungsmittelchemie, der auf dem Gebiete der Medizin erforderlichen Untersuchungen, dann daran, da $B$ G e o r $g$ A r e n d s über ein Jahrzehnt lang als wissenschaftlicher Redakteur der Pharmazeutischen Zeitung pflichtgemäß alles bearbeiten, ordnen und sichten mußte, was immer dem Apotheker auf seinem weitschichtigen Arbeitsgebiet von Nutzen sein konnte, so muß man von vornherein annehmen, daß sie der übernommenen Aufgabe nicht nur gewachsen, sondern für sie die berufenen Kräfte waren, und daß sie in ihren Mitarbeitern geeignete Helfer heranzogen. Ihr Werk bezeugt das Gesagte in jeder Beziehung. In $\mathrm{Hagers}$ Geist und nach seinem Vorbild setzten sie fort und ergänzten, was er, und nach ihm $\mathrm{F}$ is $\mathrm{ch}$ er und $\mathrm{H}$ a $\mathrm{rtw}$ i $\mathrm{ch}$ in ihrer Neubearbeitung von 1900 dem Apotheker als eine Art Vade mecum dargeboten haben. Neu bringen sie ausführliche Arbeiten über B a kteri olog i e und $S$ terilisation, über das $M$ ikroskop und seine Anwend ung, man findet ausreichende Belehrung über die $\mathrm{N}$ a h $\mathrm{r}$ u $\mathrm{n} \mathrm{s} \mathrm{m}$ i t telchemie, über Untersuchungen für $\mathbf{k} \mathbf{l} \mathbf{i}$ $\mathrm{n}$ is c h e $\mathrm{Z}$ we c k e (Harnuntersuchung usw.), die Methoden der neuen Blutunterschei d ung eine Darlegung der neuesten Forschungsergebnisse auf den Gebieten pharmazeutischer Hilfswissenschaften und nicht minder auf denen, die loser mit der Apotheke zusammenhängen, auf dem der Technik, der Kosmetik, Nah rungsmittelindustrie usw., usw. Weitschichtig, unendlich vielgestaltig ist ja das Wesen des Pharmakon, mit dem sich der Pharma. zeut $\mathrm{zu}$ beschäftigen hat, und noch weiter breitet es sich aus, seitdem die $\mathrm{T}$ i e $\mathrm{r} h$ e il $\mathbf{k}$ u $\mathrm{n} d$ e eine achtunggebietende Stellung neben der früher souveränen, nur die Menschen in Betracht ziehenden erobert. Auch sie erfährt eine umgehende Würdigung. Kaum wird der Apotheker vergeblich eine Frage an den neuen ,H a g e $\mathrm{r}^{\text {" richten. Ich }}$ habe keinen Versager bei den von mir angestellten Stichproben zu verzeichnen gehabt. Das Buch ist in der Tat einer ganzen Bibliothek an die Seite zu setzen, wie sie seit alten Zeiten dem Apotheker von dem fürsorglichen Staate als notwendige Anschaffung anbefohlen ward. Daß kleine Fehler hier und da unterlaufen sind, ist kaum zu verwundern. Wenn ich einige aufzähle, wenn ich einige Wünsche äußere, so geschieht das vielleicht auch, um nu beweisen, daß ich dem Buche die verdiente sorgfältige Prüfung habe angedeihen lassen. Die alphabetische Anordnung des Stoffes erübrigt die Aufnahme eines
Teiles der Artikel im Index. Die z. B. von A b $r u s$ war nicht nötig, die von $T r$ a descan t i a ebensowenig, aber meines Erachtens wäre z. B. zum mindesten sehr wünschenswert gewesen, H y d r a n g e a aufzunehmen, die sich unter den Fxtracta fluida verbirgt, und etwas über ihre botan. Eigenart wäre sicher auch erwünschte Beigabe. Dasselbe gilt für Fabiana (die an verschiedenen Stellen erwähnt ist) usw. Diese Unterlassungssünde fällt um so mehr auf, als $\mathrm{z}$. B. H y d r a s t is fünfmal, Y o h i m b e gar siebenmal im Index aufgeführt ist. Wenn letzteres mit $Y$ geschrieben ward, so hätte Jaborandi denselben Anfangsbuchstaben verlangen könnon. Daß auf $\mathrm{S}$. 306 viermal hintereinander Cy ripedium (im Index fehlt es auch) statt wie in der Quelle, der U.S. Pharmocopoeia richtig Cypripedi u m [der Kypris Fußbekleidung] steht, ist auffallend. Ahnlich geht es mit M e c e r e u m, das ibrigens auch im Index steht und dort mit $Z$ geschrieben ist. Gelegentlich fanden sich bei $\mathrm{H}$ a $\mathrm{g}$ e $\mathrm{r}$ kleine historische und etymologische Hinweise. Sie fehlen jetzt wohl durchweg. Die augenblickliche Generation kann ihrer entraten, im Interesse späterer wären sie äußerst erwünscht. Die Ausstattung des Buches ist eine seinem Inhalt und der Verlagshandlung entsprechend vortreffliche. Daß allerdings die Abbildung, z. B. von $R$ h i z. P a n n a e auf der Höhe steht, dürfte man nicht behaupten können. Die Hinweise auf die Arzneibücher, die einzelne Präparate, Drogen usw. aufgenommen haben, sind eine anerkennenswerte Beigabe. Ein Hinweis auf ihr Erscheinungsjahr oder eine Utbersicht der Bücher, auf die sich die Abkürzungen (z. B. Nat. Form.) beziehen, wäre doch wohl nötig gewesen. Damit möchte ich meine Besprechung schließen und nur noch die Anschaffung des Buches, ohne das selbst der kleinste Apothekenbetrieb kaum im Gange zu halten ist, und ohne das die Kreise, die irgendwie mit Pharmazie zu tun haben, schwerlich auskommen können, warm befürworten. Hermann Schelenz, Cassel.

Die Lebensvorgänge in Pflanzen und Tieren. Versuch einer Lösung der physiologischen Grundfragen von Dr. J u li u s $\mathrm{F}$ i s $\mathrm{ch}$ e $\mathrm{r}$, Ingen. Mit 13 in den Text gedruckten Figuren. Berlin, R. Friedländer \& Sohn, 1908. 83 S. M 3,Die Lebensvorgänge in Tieren und Pflanzen spielen sich nach den thermochemischen Grundgesetzen $a b$ - diesen Gedanken verfolgend, zeigt Verf. in vorliegender Schrift, wie die Abwicklung der energetischen Lebensprozesse, die Ausnutzung, die Verarbeitung der Wärme im Organismus gedacht werden kann. Er führt uns bis an die Grenze des Energetischen - da, wo der Wille (dem nach seinen Vermutungen der Impuls zu gewissen Oxydationsprozessen im Gehirn entspringen könne) in Kraft tritt, macht er Halt. Die psychischen Vorgänge können, wie im Schlußwort treffend ausgeführt wird, nicht wohl den physikalischen Gesetzen untergeordnet werden!

Für den Naturwissenschaftler im allgemeinen bieten die Ausführungen dieses hochinteressanten Themas, das Verf. eingehend in exakter Weise behandelt, gewiß eine anregende Lektüre - für den Physiologen werden sie von besonderem Werte sein! K. Kautzsch. 